\title{
A mechanically strong and ductile soft magnet with ultralow coercivity
}

\section{Dierk Raabe ( $\nabla$ d.raabe@mpie.de)}

Max-Planck-Institut für Eisenforschung https://orcid.org/0000-0003-0194-6124

\section{Liuliu Han}

Max-Planck-Institut für Eisenforschung $\mathrm{GmbH}$

\section{Fernando Fernando}

Department of Material Science, Technical University of Darmstadt

Isnaldi Souza Filho

Max-Planck-Institut für Eisenforschung https://orcid.org/0000-0002-5761-4981

\section{Nicolas Peter}

Forschungszentrum Jülich

\section{Ye Wei}

Max-Planck-Institut für Eisenforschung

\section{Baptiste Gault}

Max Planck Institute for Iron Research https://orcid.org/0000-0002-4934-0458

\section{Oliver Gutfleisch}

Department of Material Science, Technical University of Darmstadt

Zhiming Li

Central South University https://orcid.org/0000-0002-8170-5621

\section{Physical Sciences - Article}

\section{Keywords:}

Posted Date: December 10th, 2021

DOI: https://doi.org/10.21203/rs.3.rs-1145535/v1

License: (c) (1) This work is licensed under a Creative Commons Attribution 4.0 International License. Read Full License

Version of Record: A version of this preprint was published at Nature on August 10th, 2022. See the published version at https://doi.org/10.1038/s41586-022-04935-3. 


\title{
A mechanically strong and ductile soft magnet with ultralow coercivity
}

\author{
Liuliu Han ${ }^{1}$, Fernando Maccari² ${ }^{2}$, Isnaldi R. Souza Filho ${ }^{1}$, Nicolas J. Peter ${ }^{1}$, Ye Wei ${ }^{1}$, Baptiste \\ Gault $^{1}$, Oliver Gutfleisch ${ }^{2}$, Zhiming Li $^{1,3}$, Dierk Raabe ${ }^{1}$ \\ ${ }^{1}$ Max-Planck-Institut für Eisenforschung, Max-Planck-Straße 1, 40237 Düsseldorf, Germany \\ ${ }^{2}$ Department of Material Science, Technical University of Darmstadt, 64287 Darmstadt, Germany \\ ${ }^{3}$ School of Materials Science and Engineering, Central South University, 410083 Changsha, China \\ "Correspondence tolizhiming@csu.edu.cn (Z.L.), d.raabe@mpie.de (D.R.)
}

Soft magnetic materials (SMMs) are indispensable components in electrified applications and sustainable energy supply, allowing permanent magnetic flux variations in response to high frequency changes of the applied magnetic field, at lowest possible energy loss $^{1}$. The global trend towards electrification of transport, households and manufacturing leads to a massive increase in energy consumption due to hysteresis losses ${ }^{2}$. Therefore, minimizing coercivity, which scales the losses in SMMs, is crucial ${ }^{3}$. Yet, meeting this target alone is not enough: SMMs used for instance in vehicles and planes must withstand severe mechanical loads, i.e., the alloys need high strength and ductility ${ }^{4}$. This is a fundamental design challenge, as most methods that enhance strength introduce stress fields that can pin magnetic domains, thus increasing coercivity and hysteretic losses 5 . Here, we introduce a new approach to overcome this dilemma. We have designed a Fe-Co-Ni-Ta-Al multicomponent alloy with ferromagnetic matrix and paramagnetic coherent nanoparticles of well-controlled size $(\sim 91 \mathrm{~nm})$ and high volume fraction $(55 \%)$. They impede dislocation motion, enhancing strength and ductility. Yet, their small size, low coherency stress and small magnetostatic energy create an interaction volume below the magnetic domain wall width, leading to minimal domain wall pinning, thus maintaining the material's soft magnetic properties. The new material exhibits an excellent combination of mechanical and magnetic properties outperforming other multicomponent alloys and conventional SMMs. It has a tensile strength of $\sim 1336 \mathrm{MPa}$ at $54 \%$ tensile elongation, an extremely low coercivity of $\sim 78 \mathrm{~A} / \mathrm{m}(<1 \mathrm{Oe})$ and a saturation magnetization of $\sim 100 \mathrm{Am}^{2} / \mathrm{kg}$. The work opens new perspectives on developing magnetically soft and mechanically strong and ductile materials for the sustainable electrification of industry and society. 
Lowest possible coercivity is a primary goal for soft magnetic materials (SMMs), to reduce hysteresis-related energy losses, noise, heating and the associated material damage ${ }^{1-3}$. Also, new SMMs with higher strength and ductility are needed, to operate under mechanically demanding loading conditions for safety-critical parts in transportation and energy ${ }^{4}$. This multi-property profile creates a fundamental dilemma. Mechanical strength of metallic materials is produced by lattice defects and their elastic interactions with linear lattice faults that carry inelastic deformation, referred to as dislocations. However, the defects also interact with the magnetic domain walls and pin them. The loss in domain wall motion increases coercivity so that the materials lose their soft magnetic features. Therefore, current SMMs follow the design rule of avoiding lattice defects to minimize coercivity ${ }^{5}$. Vice versa, increasing an alloy's mechanical strength requires enhancing its internal stress level through defects such as dislocations, grain boundaries and precipitates ${ }^{6}$. This means that the task of making soft magnets mechanically strong is a trade-off between two mutually exclusive design strategies, namely, mechanical strength vs. unaffected domain wall motion.

The theory of the grain size dependence of coercivity ${ }^{7}$ shows its proportionality to the $6^{\text {th }}$ power of the grain size for the case of nanocrystalline materials, a relation that can be also applied to particles ${ }^{8}$. Current design of SMMs has thus focused on using small particles $(<15 \mathrm{~nm})^{8,9}$ and grain sizes $(<100 \mathrm{~nm})^{10-12}$. According to magnetic strain theory, the coercivity depends on the energy required to displace domain walls to overcome lattice barriers ${ }^{13-15}$. Here we introduce particles into multicomponent massive solid solution matrix and increase their size from the commonly used range of $5 \sim 15 \mathrm{~nm}$ to $90 \sim 100 \mathrm{~nm}$. With that, the internal stress level and the overall elastic coherency misfit energy are reduced through the smaller specific surface area (total surface area per unit of volume) of the particles, caused by the coarsening. We then propose that the particle design must follow four main rules. First, minimal pinning of domain walls requires a well-tuned and -controlled particle size distribution with optimum balance between the decrease in specific surface area and the increase in magnetostatic energy during particle coarsening. Second, the particle size must remain smaller than the domain wall width to prevent strong pinning, i.e. strong resistance against spin rotation ${ }^{6}$. Third, the particles' chemical composition and crystal structure determine their saturation magnetization; therefore, antiferromagnetic elements are usually excluded. Fourth, strengthening of the alloys is determined by the interaction between dislocations and particles and by the friction forces exerted on dislocations in the massive solid 
solution matrix. Thus, intrinsically strong intermetallic particles with minimal lattice misfit are employed. These require high forces for dislocation cutting (giving strength), but repeated cutting by ensuing dislocations emitted by the same source shear them with gradual ease along the remaining and gradually reducing particle cross sections (providing ductility).

These different mechanism considerations had next to be translated into a corresponding compositional alloy design concept. This is mainly guided by the requirement for (a) a ferromagnetic matrix with (b) high solid solution contribution, components that trigger the formation of (c) strong and stable intermetallic phases with (d) small lattice misfit relative to the matrix. These considerations have led us to the non-equiatomic iron-nickel-cobalt-tantalumaluminium ( $\mathrm{Co}_{27.7} \mathrm{Fe}_{32.6} \mathrm{Ni}_{27.7} \mathrm{Ta}_{5.0} \mathrm{Al}_{7.0}$, at.\%) multicomponent alloy (MCA). We synthesized the material in a vacuum induction melting furnace followed by conventional hot rolling and homogenization (details of the processing procedures and chemical compositions are provided in the Methods section). Through further isothermal heat treatments (1 100 h at $1173 \mathrm{~K}$ ), we obtain a wide range of particle sizes $(24 \sim 255 \mathrm{~nm})$. The particles have $\mathrm{L}_{2}$ structure and a complex composition, as presented in detail below.

Fig. 1 shows the structural characterization of the MCA with medium particle size (MMCA, where "M" stands for "medium particle size") after annealing at $1173 \mathrm{~K}$ for $5 \mathrm{~h}$. The MMCA shows an average grain size of $85.3 \pm 25.6 \mu \mathrm{m}$ (including annealing twin boundaries) according to electron backscatter diffraction (EBSD) analysis shown in Fig. 1a. The electron channelling contrast imaging (ECCI) analysis shows that the $\mathrm{L}_{2}$ particles have a high number density $\left(7.2 \times 10^{20} \mathrm{~m}^{-3}\right)$ and a large volume fraction $(\sim 55 \%)$ in homogeneous distribution within the face-centered cubic (fcc) matrix (Fig. 1b). The lattice misfit $(\sim 0.48 \%)$ between the fcc matrix and the $\mathrm{L}_{2}$ particles has been calculated using their lattice parameters ${ }^{16}$ acquired from the X-ray diffraction (XRD) patterns (Fig. 1c) by Rietveld simulation ${ }^{17}$. Such a small lattice mismatch reduces the capillary driving forces for further coarsening and the uniform dispersion prevents plastic localization at high strength ${ }^{18,19}$. The central beam dark-field (DF) transmission electron microscopy (TEM) analysis reveals that the average size of the $\mathrm{L} 1_{2}$ particles is $90.8 \pm 35.8 \mathrm{~nm}$, Fig. 1d. The corresponding selected-area electron diffraction (SAED, see inset of Fig. 1d) and high-resolution (HR)-TEM (Extended Data Fig. 1d) confirm the high coherency between the particles and the matrix. 
The elemental partitioning between the $\mathrm{L}_{2}$ precipitates and fcc solid solution matrix is characterized by atom probe tomography (APT). Fig. 1e shows the three-dimensional (3D) distribution of the volume investigated by APT, highlighted by a set of iso-surfaces delineating regions containing above 25 at.\% Fe. Fig. 1f shows the one-dimensional (1D) compositional profiles acquired along the cylinder in Fig. 1e. The profiles show that Fe partitions into the fcc matrix (36 at.\%), whereas the L12 particles are enriched in Ni (40 at.\%), Ta (13 at.\%), and Al (9 at.\%). The compositions of the fcc and $\mathrm{L}_{2}$ phases were determined by averaging over 3 APT data sets (including $10 \mathrm{~L}_{2}$ particles) as $\mathrm{Fe}_{36} \mathrm{Co}_{28} \mathrm{Ni}_{26} \mathrm{Al}_{7} \mathrm{Ta}_{3}$ and $\mathrm{Ni}_{40} \mathrm{Co}_{26} \mathrm{Ta}_{13} \mathrm{Fe}_{12} \mathrm{Al}_{9}$ (at.\%), respectively.

Besides these intragranular nanoparticles, we also observed two types of grain-boundary variants: (a) Coarse grain boundary particles $(160.2 \pm 55.3 \mathrm{~nm})$ with the same crystal structure and composition (Extended Data Fig. 1a-c) as the ones inside the grains; (b) incoherent particles with a minor fraction $(<0.3 \%)$ at triple-points of the grains with different structure (cubic Fd-3m, Fig. 1c) and composition ( $\mathrm{Ta}_{40.0} \mathrm{Co}_{26.1} \mathrm{Fe}_{20.1} \mathrm{Ni}_{11.1} \mathrm{Al}_{2.7}$, at.\%, Extended Data Fig. 1e). These two types of particles are both promoted by the high diffusion rate along the grain boundaries.
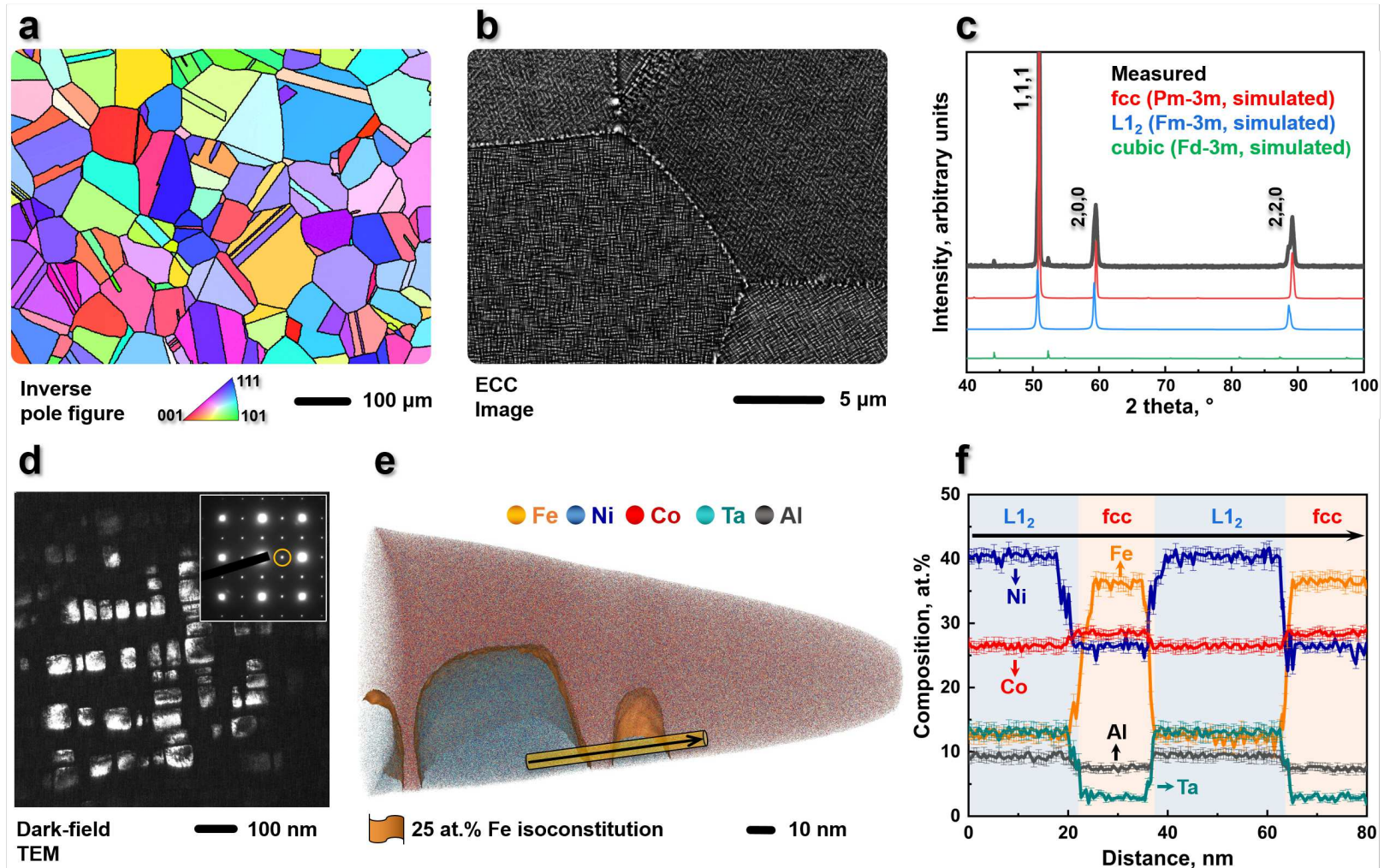

e

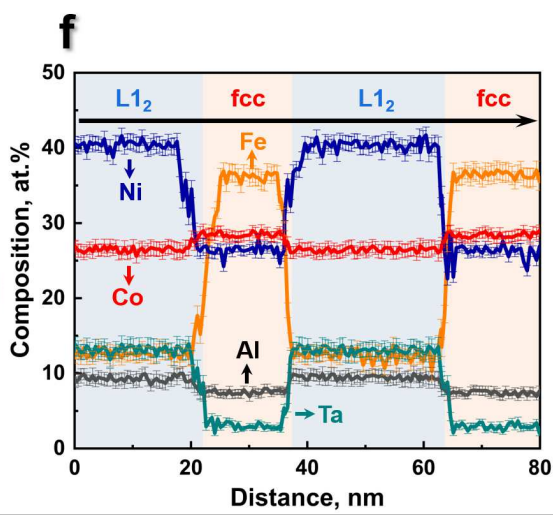


Figure 1 | Microstructure and chemical composition of the M-MCA. a, EBSD-inverse pole figure (IPF) map showing the equiaxed grains of the fcc matrix. The black lines highlight the highangle grain/twin boundaries. b, ECC image featuring the high-density uniformly distributed $\mathrm{L}_{2}$ particles in the grain interiors and heterogeneous particles at the grain boundaries. c, Measured and simulated XRD patterns showing the phase structures. d, Centered dark-field TEM image of the $\mathrm{L}_{2}$ particles obtained using the (011) superlattice spot (see inset). e, 3D reconstruction map of a typical APT tip showing the cuboidal $\mathrm{L}_{2}$ particles embedded in the fcc matrix. The $\mathrm{L} 1_{2} / \mathrm{fcc}$ interfaces are highlighted using iso-composition surfaces containing 25 at.\% Fe. f, 1D compositional profiles computed along the cylinder region (marked by black arrow) in e, showing the compositional changes across several interfaces. Error bars refer to the standard deviations of the counting statistics in each bin of the profiles.

The current strategy by tuning the particle size also enables overcoming the strengthductility trade-off, significant for advanced alloys with gigapascal-level strength. Fig. 2a displays the tensile stress-strain curves of the M-MCA at room-temperature (red curve). The yield strength $\left(\sigma_{y}\right)$ is $904 \pm 11 \mathrm{MPa}$ with an ultimate tensile strength $\left(\sigma_{\mathrm{UTS}}\right)$ of $1336 \pm 21 \mathrm{MPa}$, and an elongation at fracture $\left(\varepsilon_{\mathrm{f}}\right)$ up to $53.6 \pm 1.5 \%$, averaged from four tests. Accordingly, the M-MCA has a high $\sigma_{\mathrm{UTS}} \times \varepsilon_{\mathrm{f}}$ value of $\sim 71.6 \mathrm{GPa} \%$. To reveal the improvement in strength and ductility achieved by the well-controlled particle size distribution, the mechanical response of a material variant with identical chemical composition $\sim \mathrm{Fe}_{32} \mathrm{Co}_{28} \mathrm{Ni}_{28} \mathrm{Ta}_{5} \mathrm{Al}_{7}$ (at.\%) but smaller particle size (S-MCA, annealed for $1 \mathrm{~h}$, producing an average particle size of $\sim 24 \pm 15 \mathrm{~nm}$ ), larger particle size (L-MCA, annealed for $100 \mathrm{~h}$, average particle size of $\sim 255 \pm 49 \mathrm{~nm}$ ) and the particle-free $\mathrm{Fe}_{35} \mathrm{Co}_{30} \mathrm{Ni}_{30} \mathrm{Ta}_{5}$ (at.\%) alloy ${ }^{9}$, are also presented in Fig. 2a.

Compared with the single-phase $\mathrm{Fe}_{35} \mathrm{Co}_{30} \mathrm{Ni}_{30} \mathrm{Ta}_{5}$ (at.\%) alloy with a relatively low value of $\sigma_{y}$ of $\sim 501 \mathrm{MPa}$, the notable increase in yield strength $\left(\sigma_{y} \sim 904 \mathrm{MPa}\right)$ of the M-MCA can be attributed to the precipitation strengthening of the $\mathrm{L}_{2}$ particles with a high volume fraction ( $\left.\sim 55 \%\right)$. More importantly, such improvement in the strength of the M-MCA is achieved at no expense of ductility, which is fundamentally different from the case of the S-MCA where a significant loss in ductility is observed with increasing strength. The good ductility is correlated with the high workhardening capability, as shown in Fig. 2b. Further increase of particle size at longer annealing time $(100 \mathrm{~h})$ in the L-MCA leads to the decrease of ductility (53\%) and ultimate tensile strength (14\%) as compared to those of the M-MCA. This is related to the mechanical weakness, strain localization 
and embrittlement in the particle-free zone adjacent to the grain boundary caused by solute depletion (Extended Data Fig. 2a). This interfacial weakening has been confirmed by the associated fracture morphologies, with typical ductile fracture with fine dimples in the M-MCA material (see Fig. 2b inset) and intergranular fracture in the L-MCA material (Extended Data Fig. $2 b)$.
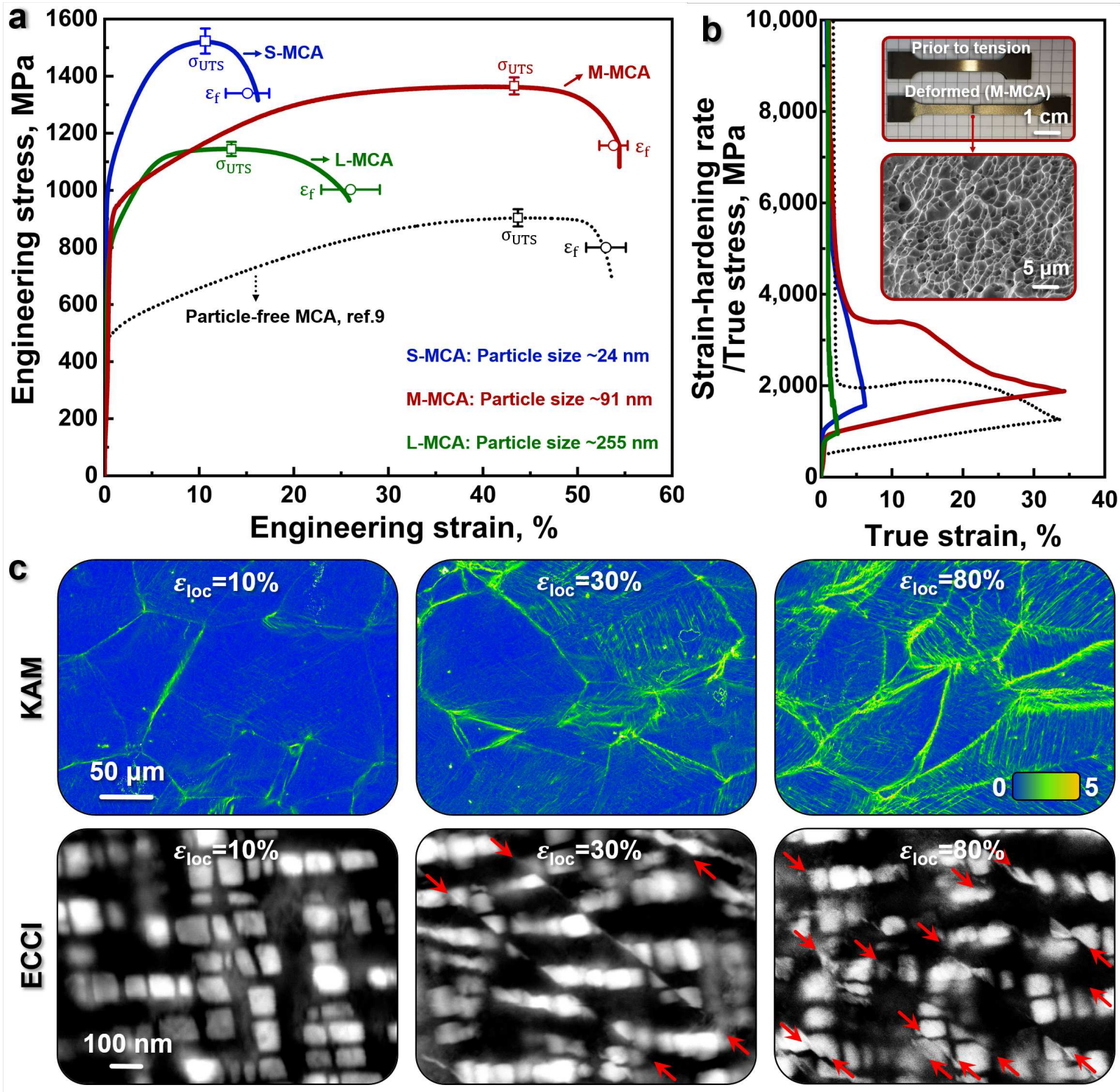

Figure 2 | Mechanical behaviour and nanoscale processes during plastic straining of the MMCA. a, Typical engineering stress-strain curves measured at room-temperature together with the average value for ultimate tensile strength $\left(\sigma_{\mathrm{UTS}}\right)$ and elongation at fracture $\left(\varepsilon_{\mathrm{f}}\right)$. b, Strain 
hardening rate/true stress-true strain curves. The insets show the macroscopic image (upper inset) of the tensile sample and corresponding fracture morphology (bottom inset), typical ductile fracture with fine dimple structures is observed. c, Substructure evolution as a function of deformation: EBSD-KAM maps revealing the deformation-induced strain distribution (upper); ECCI analysis (bottom) showing the evolution of microbands, and the shearing of $\mathrm{L}_{2}$ particles are highlighted by red arrows.

To unravel the mechanisms responsible for the significant improvement in the strengthductility combination of the M-MCA, we investigated its deformation substructures using EBSD kernel average misorientation (KAM) analysis and ECCI at different hardening stages (Fig. 2c). The underlying processes are summarized in the schematics in the Extended Data Fig. 3. In principle, high strength requires to impede dislocation movement while good ductility needs mobility of dislocations and new production of dislocations ${ }^{20}$. At the early deformation stage (local strain, $\varepsilon_{l o c}=10 \%$ ), M-MCA deforms by planar dislocation glide on $\{111\}$ planes (Extended Data Fig. 4a), as commonly observed in fcc alloys ${ }^{21}$. The dislocations extend through the grains. Corresponding pile-up configurations at the grain boundaries are revealed by the higher KAM values (Fig. 2c). The relatively large grain size $(\sim 85 \mu \mathrm{m})$ of the current M-MCA enables higher mobility of dislocations compared to most of the previously reported strong MCAs that had smaller grain $\operatorname{size}^{22-24}(\sim 10 \mu \mathrm{m})$. Further straining $\left(\varepsilon_{l o c}=30 \%\right)$ refines the crystallographically aligned microbands (Extended Data Fig. 4b). The refinement facilitates the shearing of $\mathrm{L}_{2}$ particles (see ECC images in Fig. 2c). A further refined and curved microband structure is observed at high local strains $\varepsilon_{l o c}$ of $\sim 80 \%$ (Extended Data Fig. 4c). The quantification of the evolution of the average microband spacing reveals a microband refinement process during straining (Extended Data Fig. 4d). The gradually reduced microband spacing causes a higher passing stress and thus enhanced strain hardening. This has been proposed to explain the good strength-ductility combinations in some high manganese steels $\mathrm{s}^{25-27}$ and $\mathrm{MCAs}^{28}$. Hence, the observed dynamic microband refinement and particle shearing are the prevalent deformation mechanisms in the current MCAs. No Orowan looping was observed, even when increasing the average particle size up to $255 \mathrm{~nm}$ for the L-MCA (Extended Data Fig. 2c), as the average particle spacing (Extended Data Table 1) remains far below the critical value $(\sim 3094 \mathrm{~nm}$, see Extended Data for detailed calculation) for the activation of dislocation bowing around particles, a mechanism referred to as Orowan effect. Furthermore, the 
stress required for shearing particles in the M-MCA with a medium particle size $(\sim 91 \pm 36 \mathrm{~nm})$ with high volume fraction $(\sim 55 \%)$ is 2.2 times larger than that for the S-MCA with a smaller particle size $(\sim 24 \pm 15 \mathrm{~nm})$ with low volume fraction (43\%) (see Extended Data for detailed calculation). Therefore, the high critical shear stress required for cutting the $\mathrm{L}_{2}$ particles and the dynamic microband refinement during plastic deformation lead to the strong strain hardening capacity of the M-MCA.

Fig. 3a and $\mathrm{b}$ show the magnetic properties of the MCAs. All alloys exhibit typical soft ferromagnetic behaviour. The M-MCA shows an optimal combination of an extremely low coercivity of $\sim 78 \mathrm{~A} / \mathrm{m}(<1 \mathrm{Oe})$ and a saturation magnetization $\left(M_{S}\right)$ of $\sim 100 \mathrm{Am}^{2} / \mathrm{kg}$. We identified a higher $M_{s}$ for the alloy variant with larger average particle size (see inset in Fig. 3a). The reason behind this is the change in intrinsic magnetic behaviour, as indicated by the higher Curie temperature $\left(T_{c}\right)$, revealed by the thermomagnetic curves (Extended Data Fig. 5a). Two distinct changes of the slope, indicating two ferromagnetic phases, are observed in the S-MCA. In contrast, only one sharp drop is observed in the M-MCA and L-MCA materials, indicating only one ferromagnetic phase. This is further confirmed by measuring the magnetic behaviour of the MCAs at elevated-temperature (Extended Data Fig. 5b). Considering that both, the fcc and $\mathrm{L}_{2}$ phases contain high concentrations of ferromagnetic elements, we investigated their individual magnetic response through casting both phases as separate bulk samples with their respective nominal compositions acquired before from APT analysis (see Methods for details). The results reveal that the $\mathrm{L} 1_{2}$ bulk phase is paramagnetic while the fcc matrix is ferromagnetic in the M-MCA (Extended Data Fig. 5c). Due to different partitioning, the magnetic behaviour of the $\mathrm{L}_{2}$ phase varies from ferromagnetic in the S-MCA material variant to paramagnetic in the M-MCA and L-MCA materials. The mechanism behind this transition is the change in the intrinsic spin alignment, which is related to the change in chemical composition (Extended Data Figure 6) and ordering during annealing. The overall increase in saturation magnetization of the MCAs as a function of particle coarsening is attributed to the change in fcc matrix composition due to elemental partitioning (Extended Data Fig. 6), i.e., specifically to the resultant higher concentration of (Fe+Co) in the fcc matrix. This effect enhances the total average magnetic moment per formula unit and leads to higher a $M_{s}$. 
To gain further insight into the mechanism behind the magnetic response of the M-MCA, we investigated the magnetic domain structure in terms of magneto-optical Kerr effect (MOKE) microscopy (Fig. 3c) under different applied magnetic field strengths. Starting from the alternating current (AC) demagnetized state to an applied field of $40 \mathrm{kA} / \mathrm{m}$, the nucleation of magnetic domains is uniformly distributed within the grain. Further increasing the applied field $(\sim 155 \mathrm{kA} / \mathrm{m})$ leads to domain wall movement and growth of the energetically favourable domains. The domains grow smoothly within the grains while the movement is hindered at the grain/twin boundaries (Extended Data Fig. 7). Fig. 3d summarized the statistically averaged particle size distribution with respect to the coercivity of all the MCA samples at different annealed states. The data is acquired by developing an automated processing protocol, as shown in Extended Data Fig. 8. The coercivity first decreases from $763 \mathrm{~A} / \mathrm{m}$ (S-MCA, average particle size $\sim 24 \mathrm{~nm}$ ) to $78 \mathrm{~A} / \mathrm{m}$ (M-MCA, average particle size $\sim 91 \mathrm{~nm}$ ) and then increases to $1745 \mathrm{~A} / \mathrm{m}$ (L-MCA, average particle size $\sim 255 \mathrm{~nm}$ ). Both the average particle size and the grain size increase monotonously with increasing annealing time (Extended Data Table 1). Since the grain size of the MCAs material is above the critical single-domain size, its coercivity decreases with grain coarsening, following the model for the grain size dependence of the coercivity as $H_{c} \propto 1 / D$ (where $D$ is the grain size) ${ }^{11}$. However, the magnitude of the decrease in coercivity due to grain coarsening according to the model is negligible compared to the experimentally observed values: The difference according to the model calculation between the S-MCA and M-MCA material variants is $\sim 2 \mathrm{~A} / \mathrm{m}$, yet, the experimentally observed difference is $\sim 775 \mathrm{~A} / \mathrm{m}$. Accordingly, the energy required for the irreversible displacement of domain walls within the grain is the determining effect for the extremely low coercivity. 

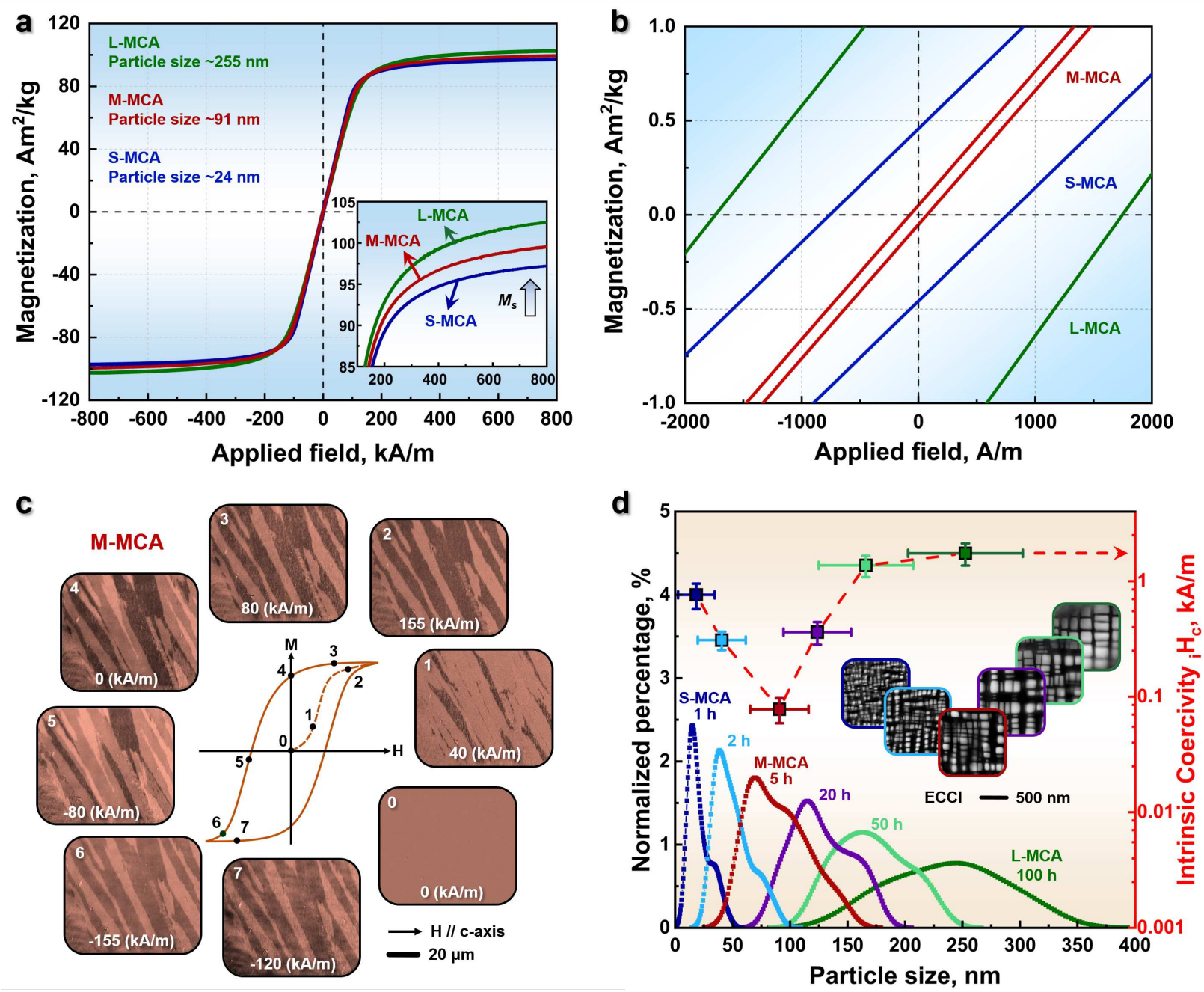

Figure 3 | Soft magnetic response and associated Bloch wall motion behaviour of the MCAs at room-temperature. a, Hysteresis loops $(M / H)$ acquired up to $\pm 800 \mathrm{kA} / \mathrm{m}$. The magnetic fieldsweeping rate is $1 \mathrm{kA} / \mathrm{m}$. The inset shows the enlarged view of the increase in saturation magnetization with particle coarsening. $\mathbf{b}, M / H$ curves measured at a rate of $0.1 \mathrm{kA} / \mathrm{m}$ between \pm $50 \mathrm{kA} / \mathrm{m}$, showing the extremely low coercivity. c, MOKE microscopy observation, in longitudinal contrast, revealing the magnetization process. The applied magnetic field is horizontal to the viewing plane. d, Statistical particle size distribution of all the MCAs under different isothermal heat treatment conditions (1 100 $\mathrm{h}$ at $1173 \mathrm{~K}$ ). The right inset shows the evolution of particle size by ECCI probing. 
Therefore, the significant drop in coercivity at the early particle coarsening stage $(\leq 91 \mathrm{~nm})$ is attributed to the gradual reduction of the overall coherency stresses between matrix and particles, due to their average size increase (see Extended Data for further calculation details). More specific, the values of the specific surface area $\times$ integrated lattice misfit decrease from $1.09 \times 10^{6} \mathrm{~m}^{-1}$ in the S-MCA material to $4.08 \times 10^{5} \mathrm{~m}^{-1}$ in the M-MCA material. The dislocation density in the matrix decreases from $1.50 \times 10^{14} \mathrm{~m}^{-2}$ in the S-MCA material to $9.32 \times 10^{13} \mathrm{~m}^{-2}$ in the M-MCA material when increasing the annealing time from $1 \mathrm{~h}$ to $5 \mathrm{~h}$ (see Extended Data for the dislocation density calculation). The reduction in dislocation density lowers the elastic distortion fields that can pin the domain walls. Although the elastic distortion and dislocation density decrease with particle coarsening, the coercivity of the L-MCA material increases. Two main mechanisms are proposed to explain this. First, the average particle size and the associated strain field in the L-MCA becomes larger than the domain wall width $\left(\delta_{w}\right)$, leading to domain wall pinning. The L-MCA has a $\delta_{w}$ of $\sim 117 \mathrm{~nm}$ and a much larger average particle size of $\sim 255 \mathrm{~nm}$, whereas the average particle size of the M-MCA is $\sim 91 \mathrm{~nm}$, which is below its $\delta_{w}$ of $\sim 112 \mathrm{~nm}$ (see Extended Data for the estimation of the domain wall width). Second, the increased magnetostatic energy $\left(E_{s}\right)$ associated with the paramagnetic particles causes a stronger individual pinning effect of each particle on the domain wall motion. More specifically, the $E_{s}$ of the L-MCA is estimated to be 23 times larger than that of the M-MCA (see Extended Data for calculation details). When only considering particle size, it should be noted that for the M-MCA, the coherent particles distributed along the grain boundaries with an average size $(\sim 160 \mathrm{~nm})$ above the $\delta_{w}(\sim 112 \mathrm{~nm})$ are expected to have a stronger pinning effect on the domain walls than those in the grain interiors $(\sim 91 \mathrm{~nm})$. However, these coarser particles only occupy a small fraction $(\sim 1.2 \%$ in M-MCA), hence, with a negligible effect on domain wall motion.

These considerations show that the nanoscale size distribution of the particles must be carefully controlled to minimize their pinning effect on domain wall movement, which determines the alloy's coercivity. This is achieved here by an optimal balance between the release of structural defects (e.g., interfacial elastic distortion, dislocation density) just down to a level required to maintain high mechanical strength and the rise of the pinning effect from the magnetostatic energy, while keeping the particle size below the domain wall width during particle coarsening. 
To highlight the pronounced combination of mechanical and soft magnetic properties of the M-MCA with optimal particle size, we compare it with existing soft magnetic materials in an Ashby-plot showing the ultimate tensile strength $\left(\sigma_{\mathrm{UTS}}\right) \times$ elongation at fracture $\left(\varepsilon_{\mathrm{f}}\right)$ against the intrinsic coercivity, Fig. 4a. This comparison shows that the $\sigma_{\mathrm{UTS}} \times \varepsilon_{\mathrm{f}}$ value of the new M-MCA material outperforms all other soft magnetic materials. More importantly, the coercivity of the new material is lower than that of all $\mathrm{NiFe}$ alloys and other MCAs, comparable only to $\mathrm{Fe}-\mathrm{Si}, \mathrm{CoFe}$ alloys and pure Fe. A similar plot showing the $\sigma_{\mathrm{UTS}} \times \varepsilon_{\mathrm{f}}$ values against the saturation induction is shown in the Extended Data Fig. 9. The comparison shows that the newly developed material with its extremely low coercivity has also a good saturation induction compared to other soft magnetic materials. Fig. $4 \mathrm{~b}$ compares the $\sigma_{\mathrm{UTS}} \times \varepsilon_{\mathrm{f}}$ value versus grain size of the M-MCA material with recently reported strong and ductile MCAs. The analysis shows that the current alloy reaches high values of $\sigma_{\mathrm{UTS}} \times \varepsilon_{\mathrm{f}}$ even without the substantial contribution from grain boundary strengthening, confirming the significant strengthening provided by the nanoparticles and the massive solid solution matrix in the current MCA.
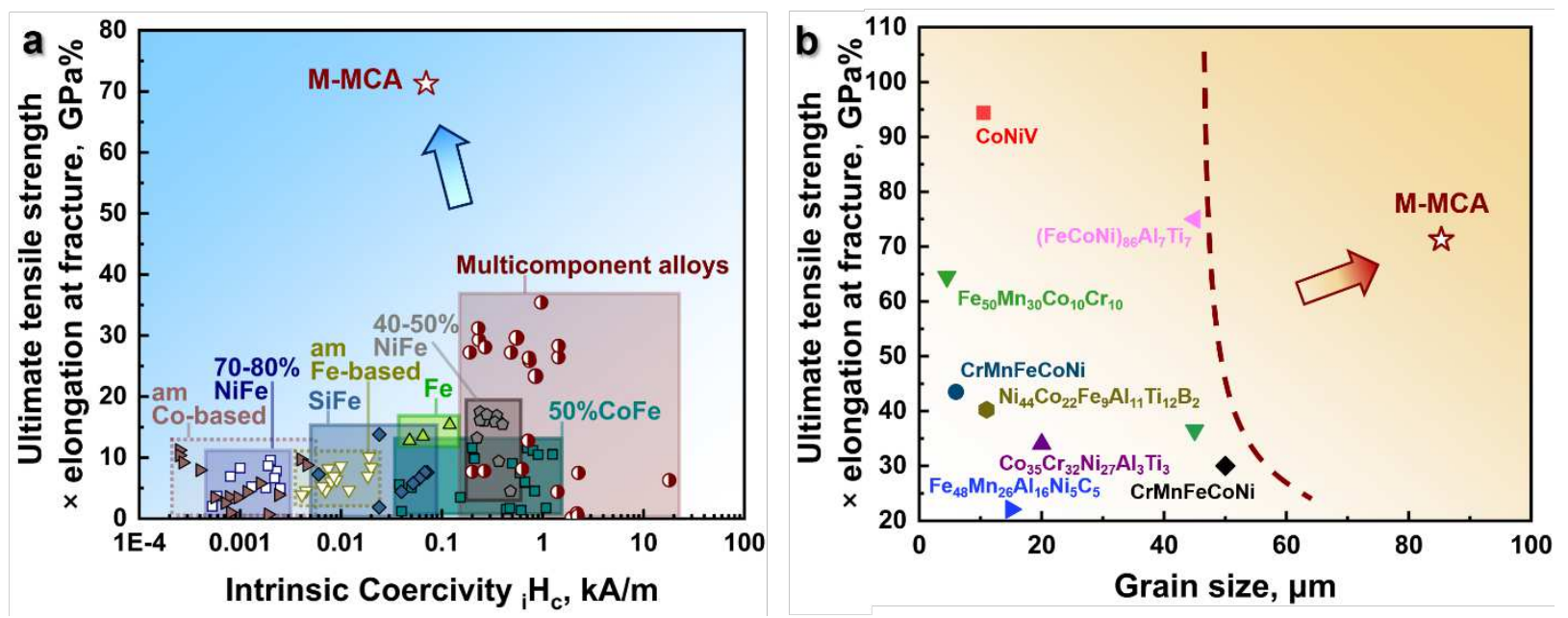

Figure 4 | Mechanical and magnetic features combined in the new $\mathrm{Fe}_{32} \mathrm{Co}_{28} \mathrm{Ni}_{28} \mathrm{Ta}_{5} \mathrm{Al}_{7}$ (at.\%) M-MCA material. a, Ashby map compiling room-temperature ultimate tensile strength $\left(\sigma_{U T S}\right) \times$ elongation at fracture $\left(\varepsilon_{\mathrm{f}}\right)$ and intrinsic coercivity compared to those of other soft magnetic materials, such as $\mathrm{NiFe}^{29,30}, \mathrm{CoFe}^{31,32}, \mathrm{SiFe}^{33}, \mathrm{Fe}^{34}$, amorphous alloys ${ }^{4,35-37}$ and established $\mathrm{MCAs}^{38-49}$. b, Ashby map showing $\sigma_{\mathrm{UTS}} \times \varepsilon_{\mathrm{f}}$ versus average grain size compared to those of other strong and ductile MCAs $\mathrm{s}^{22-24,50-53}$. 'am' in the legend stands for amorphous alloys. 
Here we have developed a new material which unifies so far mutually exclusive properties, namely, high mechanical strength (1336 MPa), high tensile ductility (54\%), extremely low coercivity $(78 \mathrm{~A} / \mathrm{m})$ and good saturation magnetization $\left(100 \mathrm{Am}^{2} / \mathrm{kg}\right)$. We realized this in a new class of bulk SMMs through a nanoparticle dispersion with well-controlled size $(\sim 91 \mathrm{~nm})$, magnetic properties, coherency strain, strength and interface energy. The design strategy is opposite to that generally applied in conventional SMM design. Instead of using smallest microstructure features (particle size $<15 \mathrm{~nm}$ ) to avoid magnetic wall pinning as in conventional SMMs, we have chosen a relatively coarse particle dispersion with tuned particle/matrix interfacial coherency stresses and paramagnetic properties to minimize magnetic pinning of domain walls on the one hand (soft magnetism) and maximize interaction strength with dislocations on the other hand (strength and ductility). Such a particle design concept can be applied also to many other precipitation-containing soft magnetic alloys, suited to serve in a broad spectrum of mechanicallydemanding energy, automotive and aerospace applications. 


\section{References}

1 J. M. Silveyra, et al. Science 362, eaao0195 (2018). https://doi.org/10.1126/science.aao0195

2 S. Velayos, V. Sanchez-Giron, Nature 157, 583 (1946). https://doi.org/10.1038/157583b0

3 O. Gutfleisch, et al. Adv. Mater. 23, 821-842 (2011). https://doi.org/10.1002/adma.201002180

A. Inoue, et al. Nat. Mater. 2, 661-663 (2003). https://doi.org/10.1038/nmat982

T. Hasegawa, et al. Sci. Rep. 7, 13125 (2017). https://doi.org/10.1038/s41598-017-13602-x

B. D. Cullity, C. D. Graham, Introduction to Magnetic Materials (IEEE Press, ed. 2nd, 2009). htt ps://doi.org/10.1002/9780470386323

Z. Fu, et al. Appl. Mater. Today, 15, 590-598 (2019). https://doi.org/10.1016/j.apmt.2019.04.014

G. Herzer, IEEE Trans. Magn. 25, 3327-3329 (1989). https://doi.org/10.1109/20.42292

T. Osaka, et al. Nature 392, 796-798 (1998). https://doi.org/10.1038/33888

L. Landau, E. Lifshitz, Phys. Z. 8, 153-164 (1935). https://doi.org/10.1016/B978-0-08-0363646.50008-9

14 L. Néel, Cah. Phys. 25, 1-20 (1944).

15 S. Chikazumi, C. D. Graham, Physics of Ferromagnetism. (Oxford University, 2012). ISBN: 9783-642-25582-3

16 A. G. Khachaturyan, Theory of Structural Transformations in Solids (Courier Corporation, 2013). ISBN: 978-0-486-46280-6

17 R. J. Hill, C. J. Howard, J. Appl. Cryst. 20, 467-474 (1987). https://doi.org/10.1107/S0021889887 086199

18 S. Jiang, et al. Nature 544, 460-464 (2017). https://doi.org/10.1038/nature22032

19 T. Yang, et al. Science 362, 933-937 (2018). https://doi.org/10.1126/science.aas8815

S. Tolansky, Nature 209, 1058-1059 (1966). https://doi.org/10.1038/2091058b0

C. S. Pande, P. M. Hazzledine, Philos. Mag. 24, 1393-1410 (1971).

T. Yang, et al. Science 369, 427-432 (2020). https://doi.org/10.1126/science.abb6830

H. Luo, et al. Nat Commun 11, 3081 (2020). https://doi.org/10.1038/s41467-020-16791-8

Z. Wang, et al. Sci. Adv. 6, eaba9543 (2020). https://doi.org/doi:10.1126/sciadv.aba9543

E. Welsch, et al. Acta Mater. 116, 188-199 (2016). https://doi.org/10.1016/j.actamat.2016.06.037

M. Yao, et al. Acta Mater. 140, 258-273 (2017). https://doi.org/10.1016/j.actamat.2017.08.049

Z. Wang, et al. Acta Mater. 198, 258-270 (2020). https://doi.org/10.1016/j.actamat.2020.08.003

Z. Wang, et al. Acta Mater. 126, 346-360 (2017). https://doi.org/10.1016/j.actamat.2016.12.074

T. R. Christenson, et al. MRS Online Proc. Libr. 518, 185-190 (1998). https://doi.org/10.1557/PR OC-518-185

30 P. V. Yekta, et al. J. Magn. Magn. Mater. 468, 155-163 (2018). https://doi.org/10.1016/j.jmmm.2 018.07 .088

31 E. P. George, et al. Mater. Sci. Eng., A 329, 325-333 (2002). https://doi.org/10.1016/S09215093(01)01594-5

32 R. M. Bozorth, Ferromagnetism. (Wiley, 1993). ISBN: 978-0-780-31032-2

33 C. W. Chen, Magnetism and metallurgy of soft magnetic materials (Courier Corporation, 2013). ISBN-0-486-64997-0

34 W. Pepperhoff, M. Acet, Constitution and Magnetism of Iron and its Alloys. (Springer, 2013). ISBN 978-3-662-04345-5

35 A. Inoue, et al. Acta Mater. 52, 1631-1637 (2004). https://doi.org/10.1016/j.actamat.2003.12.008

36 C. Y. Lin, et al. J. Phys. D: Appl. Phys. 40, 310-314 (2007). https://doi.org/10.1088/00223727/40/2/004

37 B. Shen, et al. Appl. Phys. Lett. 85, 4911-4913 (2004). https://doi.org/10.1063/1.1827349 
P. Li, et al. J. Alloys Compd. 694, 55-60 (2017). https://doi.org/10.1016/j.jallcom.2016.09.186

41 T. T. Zuo, et al. J. Magn. Magn. Mater. 371, 60-68 (2014). https://doi.org/10.1016/j.jmmm.2014. 07.023

42 Y. P. Wang, et al. Adv. Eng. Mater. 11, 641-644 (2009). https://doi.org/10.1002/adem.200900057

43 K. B. Zhang, et al. J. Alloys Compd. 502, 295-299, (2010). https://doi.org/10.1016/j.jallcom.2009. 11.104

44 S. G. Ma, Y. Zhang, Mater. Sci. Eng., A 532, 480-486 (2012). https://doi.org/10.1016/j.msea.201 $\underline{1.10 .110}$

45 K. Zhang, Z. Fu, Intermetallics 28, 34-39 (2012). https://doi.org/10.1016/j.intermet.2012.03.059

46 S. M. Na, et al. AIP Advances 8, 056412 (2018). https://doi.org/10.1063/1.5007073

47 H. Duan, et al. Mater. Res. Bull. 111, 289-293 (2019). https://doi.org/10.1016/j.materresbull.201 $\underline{8.11 .036}$

48 H. Zhang, et al. J. Magn. Magn. Mater.478, 116-121 (2019). https://doi.org/10.1016/j.jmmm.201 $\underline{9.01 .096}$

49 T. Zuo, et al. Intermetallics 100, 1-8 (2018). https://doi.org/10.1016/j.intermet.2018.05.014

50 Z. Li, et al. Nature 534, 227-230 (2016). https://doi.org/10.1038/nature17981

51 X. H. Du, et al. Nat Commun 11, 2390 (2020). https://doi.org/10.1038/s41467-020-16085-Z

52 B. Gludovatz, et al. Science 345, 1153-1158 (2014). https://doi.org/10.1126/science.1254581

53 Z. Wu, et al. Acta Mater. 81, 428-441 (2014). https://doi.org/10.1016/j.actamat.2014.08.026

\section{Methods}

Materials preparation. The bulk MCA ingot with a predetermined nominal composition of $\mathrm{Co}_{27.7} \mathrm{Fe}_{32.6} \mathrm{Ni}_{27.7} \mathrm{Ta}_{5.0} \mathrm{Al}_{7.0}$ (at\%) was first cast in a vacuum induction furnace using pure metallic ingredients (purity higher than $99.8 \mathrm{wt} . \%$ ) under high-purity argon (Ar) atmosphere. The as-cast ingot with a dimension of $40 \mathrm{~mm} \times 60 \mathrm{~mm} \times 20 \mathrm{~mm}($ length $\times$ width $\times$ thickness $)$ was then hot-rolled at $1473 \mathrm{~K}$ to an engineering thickness reduction of $50 \%$ (final thickness $\sim 10 \mathrm{~mm}$ ). After hot-rolling, The alloy sheets were then homogenized at $1473 \mathrm{~K}$ for $10 \mathrm{~min}$ in Ar atmosphere followed by water-quenching. To obtain a wide size distribution of the particles, further isothermal heat treatments were conducted at $1173 \mathrm{~K}$, lasting from $1 \mathrm{~h}$ up to $100 \mathrm{~h}(1 \mathrm{~h}, 2 \mathrm{~h}, 5 \mathrm{~h}, 20 \mathrm{~h}, 50 \mathrm{~h}, 100$ h) in Ar atmosphere and followed by water quenching. The exact chemical composition of the MCA measured by wet-chemical analysis is $\mathrm{Co}_{28.0} \mathrm{Fe}_{32.0} \mathrm{Ni}_{28.1} \mathrm{Ta}_{4.7} \mathrm{Al}_{7.2}$ (at.\%), which is close to the pre-designed composition. In addition, the bulk ingots $(\sim 50 \mathrm{~g})$ with the compositions identical to that of the fcc $\left(\mathrm{Fe}_{36} \mathrm{Co}_{28} \mathrm{Ni}_{26} \mathrm{Al}_{7} \mathrm{Ta}_{3}\right.$, at\%) matrix phase and the $\mathrm{L}_{2}$ particle $\left(\mathrm{Ni}_{40} \mathrm{Co}_{26} \mathrm{Ta}_{13} \mathrm{Fe}_{12} \mathrm{Al}_{9}\right.$, at.\%) in the M-MCA derived from the APT analysis were also cast, respectively, by arc-melting under Ar atmosphere. The ingots were remelted six times to achieve chemical homogeneity. 
Analytical methods. X-ray diffraction (XRD) measurements were carried out in an X-ray system (Diffractometer D8 Advance A25-X1) with Co K $\alpha$ radiation $(\lambda=1.78897 \AA$, $35 \mathrm{kV}$ and $40 \mathrm{~mA}$ ). Electron backscatter diffraction (EBSD) characterizations were conducted in a Zeiss-Crossbeam focus ion beam scanning electron microscope at $15 \mathrm{kV}$. Electron channelling contrast imaging (ECCI) characterizations were performed using a Zeiss-Merlin high-resolution field emission electron microscope at $30 \mathrm{kV}$. Transmission electron microscopy (TEM) analysis including selected area electron diffraction (SAED) was conducted in a JEOL JEM 2100 at $200 \mathrm{kV}$. Scanning transmission electron microscopy (STEM) images were collected using a probe-corrected Titan Themis 60-300 (Thermo Fischer Scientific) microscope. To modify the Z-contrast characteristics of the imaging mode, high-angle annular dark-field (HAADF) micrographs with a convergence angle of $23.8 \mathrm{mrad}$ were acquired at $300 \mathrm{kV}$. The resulting collection angle ranges from $73 \mathrm{mrad}$ to $200 \mathrm{mrad}$. Further energy-dispersive X-ray spectroscopy (EDS) analysis was conducted using Thermo Fischer Scientific's Super-X windowless EDS detector at an acceleration voltage of 300 $\mathrm{kV}$. Atom probe tomography (APT) experiments were performed in a local electrode atom probe (LEAP 5000 XR) from Cameca Instruments Inc and analyzed with commercial AP Suite software (v 6.1). A pulse frequency of $\sim 125 \mathrm{kHz}$, a pulse energy of $\sim 40 \mathrm{pJ}$, and a temperature of $\sim 60 \mathrm{~K}$ was used. The detection rate was kept at a frequency of 1 ion per 100 pulses.

Mechanical response measurements. Room-temperature uniaxial tensile tests were performed using flat tensile specimens at an initial strain rate of $1 \times 10^{-3} \mathrm{~s}^{-1}$. The tensile specimens in twogauge dimensions were machined along the rolling direction from the alloy sheets by electrical discharge machining. The specimens with a total length of $60 \mathrm{~mm}$, a gauge length of $30 \mathrm{~mm}$, a gauge width of $5 \mathrm{~mm}$ and a thickness of $2 \mathrm{~mm}$ were used to probe the bulk tensile properties. Further, smaller tensile specimens with a total length of $20 \mathrm{~mm}$, a gauge length of $10 \mathrm{~mm}$, a gauge width of $2 \mathrm{~mm}$ and a thickness of $1 \mathrm{~mm}$ were used to measure the local strain evolution by the digital image correlation (DIC) method. At least four specimens for each condition were tested to confirm reproducibility.

Magnetic response measurements. The magnetic response was evaluated using the Quantum Design Magnetic Properties Measurement System (MPMS) equipped with a standard vibrating sample magnetometry (VSM) option. Cuboid specimens in $\sim 3 \mathrm{~mm} \times 3 \mathrm{~mm} \times 1 \mathrm{~mm}$ (length $\times$ width $\times$ thickness) were used for the measurements. The hysteresis loops $M(H)$ were 
performed in an external magnetic field of $\pm 800 \mathrm{kA} / \mathrm{m}$ at a magnetic field-sweeping rate of $1 \mathrm{kA} / \mathrm{m}$ at $10 \mathrm{~K}, 300 \mathrm{~K}, 500 \mathrm{~K}$ and $800 \mathrm{~K}$, respectively. The temperature dependence of magnetization $M(T)$ analysis was carried out under an applied field of $40 \mathrm{kA} / \mathrm{m}$ from $10 \mathrm{~K}$ to $1000 \mathrm{~K}$ with a temperature-sweeping rate of $10 \mathrm{~K} / \mathrm{min}$.

The magnetic domain patterns were characterized by a magneto-optical Kerr effect (MOKE) Zeiss microscope (Axio Imager.D2m). The domain wall movement was captured under an applied magnetic field of $\pm 155 \mathrm{kA} / \mathrm{m}$. Prior to the measurement, a background image was collected as a reference in the alternating current (AC) demagnetized state. The images acquired at different applied fields were enhanced by subtracting the background image using KerrLab software.

\section{Estimation of interfacial coherency stress}

The coherency stress at the $\mathrm{L} 1_{2} / \mathrm{fcc}$ interface is determined by integrating the lattice misfit across the interface as:

$$
\delta=S_{\mathrm{L} 1_{2} / \text { fcc }} \sum \delta_{x}
$$

where $S_{\mathrm{L}_{2} / \mathrm{fcc}}$ is the $\mathrm{L} 1_{2} /$ fcc interface area related to the average particle size $(d)$, the volume fraction of the $\mathrm{L} 1_{2}$ particles $(f)$ and the overall volume $(V)$ as follows:

$$
S_{\mathrm{L} 1_{2} / \mathrm{fcc}}=\frac{V f}{4 / 3 \pi \cdot\left(\frac{d}{2}\right)^{3}} 4 \pi(d / 2)^{2}=\frac{6 V f}{d}
$$

$\delta_{x}$ is the varying lattice misfit as a function of distance $(x)$ from the $\mathrm{L} 1_{2} /$ fcc interface determined by the following equation ${ }^{16}$ :

$$
\delta_{x}=2 \times\left[\frac{a_{x}^{\mathrm{L} 12}-a_{x}^{\mathrm{fcc}}}{a_{x}^{\mathrm{L} 12}+a_{x}^{\mathrm{fcc}}}\right]
$$

$a_{x}^{\mathrm{L} 1_{2}}$ and $a_{x}^{\mathrm{fcc}}$ are the lattice parameters of the $\mathrm{L} 1_{2}$ and fcc phases at the interfacial region. Such values were calculated using the $\mathrm{L}_{2} /$ fcc interfacial chemical compositions acquired from the APT datasets with Vegard's relation ${ }^{54}$ :

$$
\begin{gathered}
a_{x}^{\mathrm{L} 1_{2}}=a_{0}^{\mathrm{L} 1_{2}}+\sum_{i} \Gamma_{i}^{\mathrm{L} 1_{2}} x_{i}^{\mathrm{L} 1_{2}} \\
a_{x}^{\mathrm{fcc}}=a_{0}^{\mathrm{fcc}}+\sum_{i} \Gamma_{i}^{\mathrm{fcc}} x_{i}^{\mathrm{fcc}}
\end{gathered}
$$


where $a_{0}^{\mathrm{L} 1_{2}}$ and $a_{0}^{\mathrm{fcc}}$ are the average lattice parameters for the $\mathrm{L} 1_{2}$ particles and the fcc matrix, respectively, derived from the Rietveld simulation based on the XRD measurements, as shown in Extended Data Table 1. $\Gamma_{i}^{\mathrm{L} 1_{2}}$ and $\Gamma_{i}^{\mathrm{fcc}}$ are the Vegard coefficients for the $\mathrm{L}_{2}$ and fcc phases obtained from the ordered $\mathrm{Ni}_{3} \mathrm{Al}$ phase and disordered fcc phase in the Ni-base superalloys ${ }^{55}$, as shown in Extended Data Table 2. Note that the above-calculated lattice misfit $\delta_{l}$ represents the theoretical unconstrained state. This can be related to the constrained misfit $(\varepsilon)$ by elasticity theory as below ${ }^{56}$ :

$$
\varepsilon=\frac{3}{2} \delta_{l}
$$

The estimated interfacial constrained misfit value is $1.09 \times 10^{6}, 4.08 \times 10^{5}$ and $1.96 \times 10^{5}$ for the S-MCA, M-MCA and L-MCA, respectively. Therefore, the significant decrease in the interfacial coherency stress is expected to play an essential role in releasing the pining effect on domain wall movement with particle coarsening for the MCAs with the particle size below the domain wall width.

\section{Estimation of dislocation density}

The dislocation density $(\rho)$ in the fcc matrix can be calculated through the WilliamsonSmallman relationship as ${ }^{57}$ :

$$
\rho=\frac{2 \sqrt{3}\left(\varepsilon_{s}{ }^{2}\right)^{1 / 2}}{D b}
$$

where $\varepsilon_{s}$ is microstrain, $D$ is crystallite size acquired from the XRD profiles (Extended Data Table 1 ), and $b$ is the Burgers vector (for fcc structure, $b=\sqrt{2} / 2 \times a_{\mathrm{fcc}}$ ) 58 . The dislocation density in the fcc matrix is thus estimated to be $1.50 \times 10^{14} \mathrm{~m}^{-2}, 9.32 \times 10^{13} \mathrm{~m}^{-2}$ and $5.38 \times 10^{13} \mathrm{~m}^{-2}$ for the S-MCA, M-MCA and L-MCA, respectively. Based on the above estimation, the considerable improvement in the coercivity also derives from the decrease of dislocation density in the fcc matrix. 


\section{Estimation of particle shearing stress}

Based on the experimental observation (Fig. 2c and Extended Data Fig. 2c), particle shearing is the primary deformation mechanism in the investigated MCAs. The strengthening contribution of particle shearing $(\Delta \tau)$ is estimated according to ${ }^{59}$ :

$$
\Delta \tau_{\text {Shearing }}=\frac{F}{b \cdot 2 \lambda}
$$

where $2 \lambda$ is the mean spacing of the particles, $2 \lambda \approx \sqrt{\frac{2}{f}} r, r$ is the average radius of the particles $(r=d / 2) . f$ is the volume fraction of the particles shown in Extended Data Table 1, $F$ is the force exerted on the particles. The shearing strength is expressed as:

$$
\Delta \tau_{\text {Shearing }}=\mathrm{k} \sqrt{f r}
$$

by using the relation $F \propto r^{3 / 2}$ and introducing constant $\mathrm{k}$. The effect of particle strengthening of the M-MCA is then estimated to be two times larger than that of the S-MCA $\left(\Delta \tau_{M-M C A} /\right.$ $\left.\Delta \tau_{S-M C A}=\frac{\mathrm{k} \sqrt{f_{M-M C A} \cdot r_{M-M C A}}}{\mathrm{k} \sqrt{f_{S-M C A} \cdot r_{S-M C A}}}\right)$.

When considering the volume fraction of the particles to be constant, the mean spacing of the particles increases with increasing the particle size. As a result, the force required for shearing particles increases until the Orowan mechanism is activated, i.e., dislocations bowing the particles becomes easier than shearing. The critical mean spacing of the particles is determined by ${ }^{59}$ :

$$
\Delta \tau_{\text {Shearing }}=\frac{F}{b \cdot 2 \lambda}=\Delta \tau_{\text {Orowan }}=\frac{\mathrm{G} b}{2 \lambda}
$$

$\mathrm{G}=84 \mathrm{GPa}$ is the adopted shear modulus ${ }^{60}$. Consequently, the critical mean spacing of the particles is calculated as $3094.3 \mathrm{~nm}$.

\section{Estimation of magnetostatic energy}

The magnetostatic energy $\left(E_{S}\right)$ determines the coercive force which interacting between the paramagnetic particles (for M-MCA and L-MCA) and domain wall movement according to the formula ${ }^{33}$ :

$$
E_{s}=\frac{1}{2} \mu_{0} \frac{1}{3} M_{s}^{2} \frac{4}{3} \pi r^{3}
$$


where $\mu_{0}=4 \pi \times 10^{-7} \mathrm{H} / \mathrm{m}$ is the permeability of vacuum, $r$ is the average particle radius, $M_{s}$ is the saturation magnetization of the fcc matrix. For M-MCA and L-MCA, in which the $\mathrm{L}_{2}$ phase is paramagnetic (Extended Data Fig. 5), the $M_{s}$ of the fcc matrix is considered as the overall $M_{S}$ of the alloy. The values of $E_{S}$ significantly increase with increasing the particle size, that is, it varies from $6.5 \times 10^{-24}$ (M-MCA) to $1.53 \times 10^{-22}$ (L-MCA). The notable increase in magnetostatic energy results in a strong magnetic pinning effect.

\section{Estimation of domain wall width}

Strong pinning arises and results in the deterioration of coercivity when the microstructure defects have a comparable dimension to the domain wall thickness $\left(\delta_{w}\right)$. As a result, the estimation of the $\delta_{w}$ helps understand the ultralow coercivity in the current work is given by ${ }^{61,62}$ :

$$
\delta_{w}=\pi\left(A_{e x} / \mathrm{K}_{1}\right)^{1 / 2}
$$

where $A_{e x}=\mathrm{k}_{\mathrm{B}} T_{c} / 2 a_{0}$ is the exchange stiffness, $\mathrm{k}_{\mathrm{B}}=1.380649 \times 10^{-23} \mathrm{~J} / \mathrm{K}$ is the Boltzmann's constant, $T_{c}$ and $a_{0}$ are the Curie temperature and lattice parameter of the fcc matrix, respectively (Extended Data Fig. $5 d$ and Extended Data Table 1). $K_{1}$ is the first magnetocrystalline anisotropy constant. The value of $\mathrm{K}_{1}$ (M-MCA) is taken from the Co-Fe system ${ }^{63,64}$ based on the composition of the fcc matrix (Fig. 1f) as $10.4 \mathrm{~kJ} / \mathrm{m}^{3}$ ( $\mathrm{Al}$ and Ta are non-ferromagnetic elements that do not show any magnetic moment, the chemical composition of the fcc phase $\sim \mathrm{Fe}_{36} \mathrm{Co}_{28} \mathrm{Ni}_{26} \mathrm{Al}_{7} \mathrm{Ta}_{3}$, at.\%, in the M-MCA is thus considered as $\sim \mathrm{Co}_{31}(\mathrm{Fe}+\mathrm{Ni})_{69}$, at.\%). The domain wall thickness of the $\mathrm{M}-$ MCA is thus estimated to be $\sim 112 \mathrm{~nm}$. Similarly, the domain wall thicknesses of the S-MCA and L-MCA are calculated as $\sim 103 \mathrm{~nm}$ and $117 \mathrm{~nm}$, respectively. 


\section{References}

54 Y. Mishima, et al. Acta Metall. 33, 1161-1169 (1985). https://doi.org/10.1016/00016160(85)90211-1

55 H. Okamoto, et al. Ni (Nickel) Binary Alloy Phase Diagrams (ASM International, 1991) https://doi.org/10.31399/asm.hb.v03.a0006189

56 E. Nembach, G. Neite, Prog. Mater. Sci. 29, 177-319 (1985). https://doi.org/10.1016/00796425(85)90001-5

57 G. K. Williamson, W. H. Hall, Acta Metall. 1, 22-31 (1953). https://doi.org/10.1016/00016160(53)90006-6

58 W. D. Callister Jr, D. G. Rethwisch, Fundamentals of materials science and engineering: an integrated approach. (Wiley, 2018). ISBN: 978-1-119-17550-6

59 J. Roesler, et al. Mechanical behaviour of engineering materials: metals, ceramics, polymers, and composites. (Springer, 1st ed., New York, 2007).

60 Z. Wu, et al. Acta Mater. 81, 428-441 (2014). https://doi.org/10.1016/j.actamat.2014.08.026

61 L. Néel, Ann. Univ. Grenoble. 22, 299-343 (1946). http://www.numdam.org/item?id=AUG_1946 $22 \quad 299 \quad 0$

62 F. Pfeifer, C. Radeloff, J. Magn. Magn. Mater. 19, 190-207 (1980). https://doi.org/10.1016/0304$\underline{8853(80) 90592-2}$

63 L. W. McKeehan, Phys. Rev. 51, 136-139 (1937). https://doi.org/10.1103/PhysRev.51.136

64 J. W. Shin, Phys. Rev. 46, 139-142 (1934). https://doi.org/10.1103/PhysRev.46.139

\section{Acknowledgements}

The support of Prof. Gerhard Dehm at the Max-Planck-lnstitut für Eisenforschung is gratefully acknowledged. L. Han would like to acknowledge the financial support from the China Scholarship Council (201906370028). Z. Li. would like to acknowledge the National Natural Science Foundation of China (51971248) and the Hunan Special Funding for the Construction of Innovative Province (2019RS1001). O. Gutfleisch would like to acknowledge the Deutsche Forschungsgemeinschaft (405553726-TRR 270).

\section{Author contributions}

L. Han, Z. Li and D. Raabe designed the research project; L. Han, N. Peter and F. Maccari characterized the alloys; L. Han, I. Souza Filho, Y. Wei and B. Gault analyzed the data; L. Han, Z. Li, O. Gutfleisch and D. Raabe conceptualized the paper; all authors contributed to the discussion of the results.

\section{Completing interests}

The authors declare no competing interests. 


\section{Author Information}

Reprints and permissions information is available at www.nature.com/reprints. The authors declare no competing financial interests. Readers are welcome to comment on the online version of the paper. Correspondence and requests for materials should be addressed to D. Raabe (d.raabe@mpie.de) or Z. Li (lizhiming@csu.edu.cn). 


\section{Figures}

a
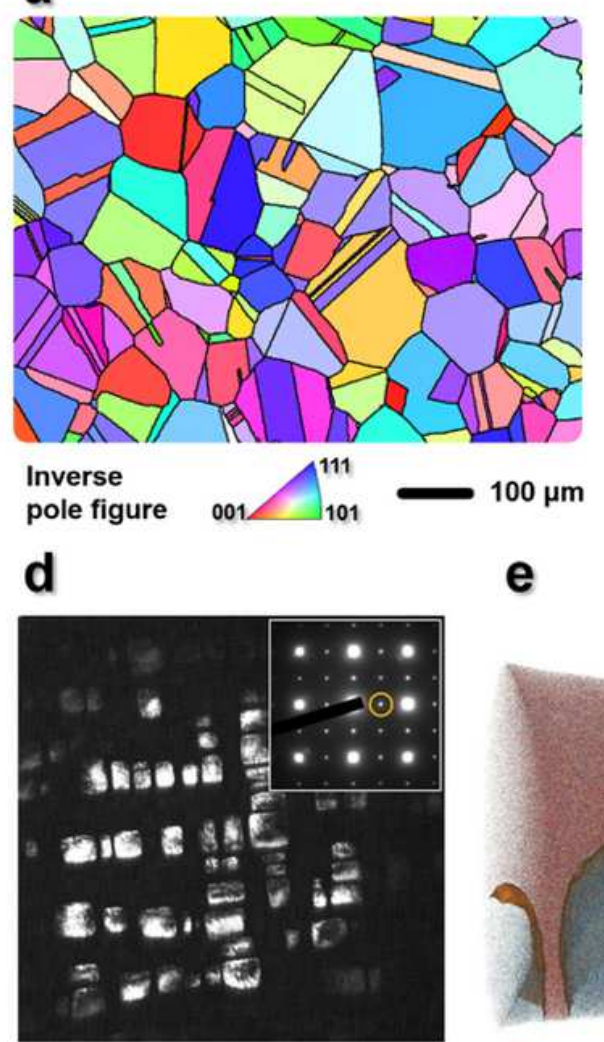

Dark-field TEM b

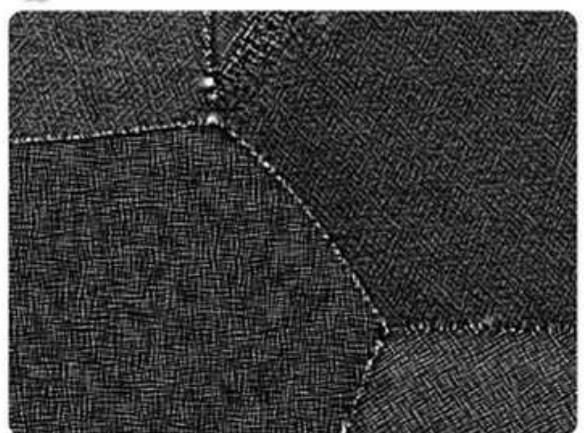

ECC

Image

$5 \mu \mathrm{m}$

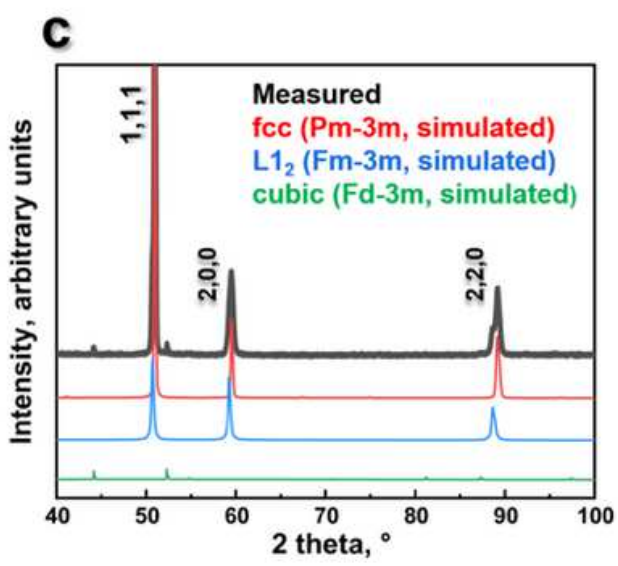

- Fe $\bigcirc \mathrm{Ni} \odot \mathrm{Co} \bigcirc \mathrm{Ta} \bigcirc \mathrm{Al}$

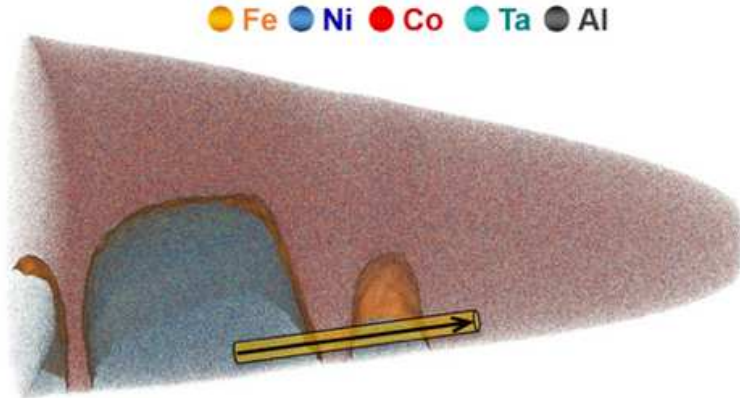

25 at.\% $\mathrm{Fe}$ isoconstitution
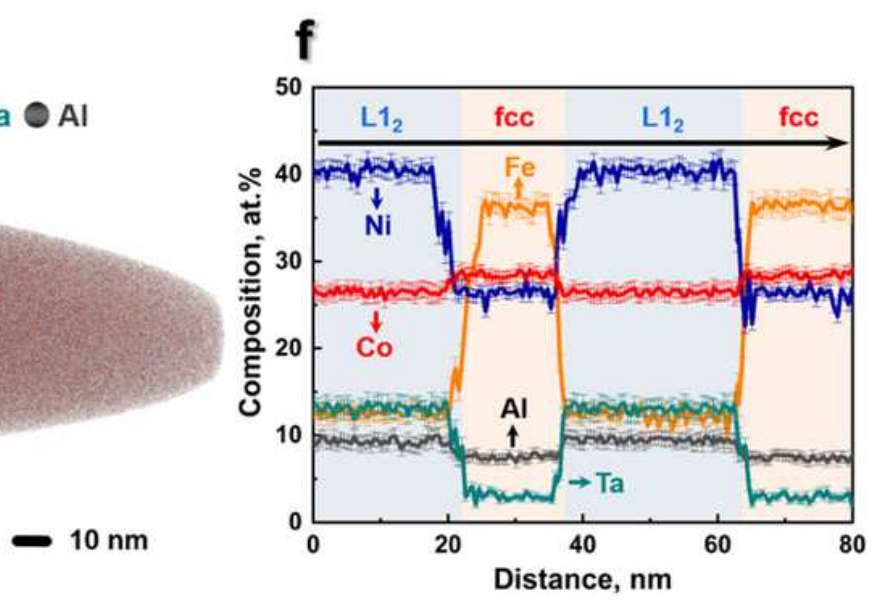

\section{Figure 1}

Microstructure and chemical composition of the M-MCA. a, EBSD-inverse pole figure (IPF) map showing the equiaxed grains of the $\mathrm{fcc}$ matrix. The black lines highlight the high-angle grain/twin boundaries. $b$, ECC image featuring the high-density uniformly distributed L12 particles in the grain interiors and heterogeneous particles at the grain boundaries. $c$, Measured and simulated XRD patterns showing the phase structures. $d$, Centered dark-field TEM image of the L12 particles obtained using the (011) superlattice spot (see inset). e, 3D reconstruction map of a typical APT tip showing the cuboidal L12 particles embedded in the fcc matrix. The $\mathrm{L} 12 / \mathrm{fcc}$ interfaces are highlighted using iso-composition surfaces containing 25 at.\% Fe. f, 1D compositional profiles computed along the cylinder region (marked by black arrow) in e, showing the compositional changes across several interfaces. Error bars refer to the standard deviations of the counting statistics in each bin of the profiles. 

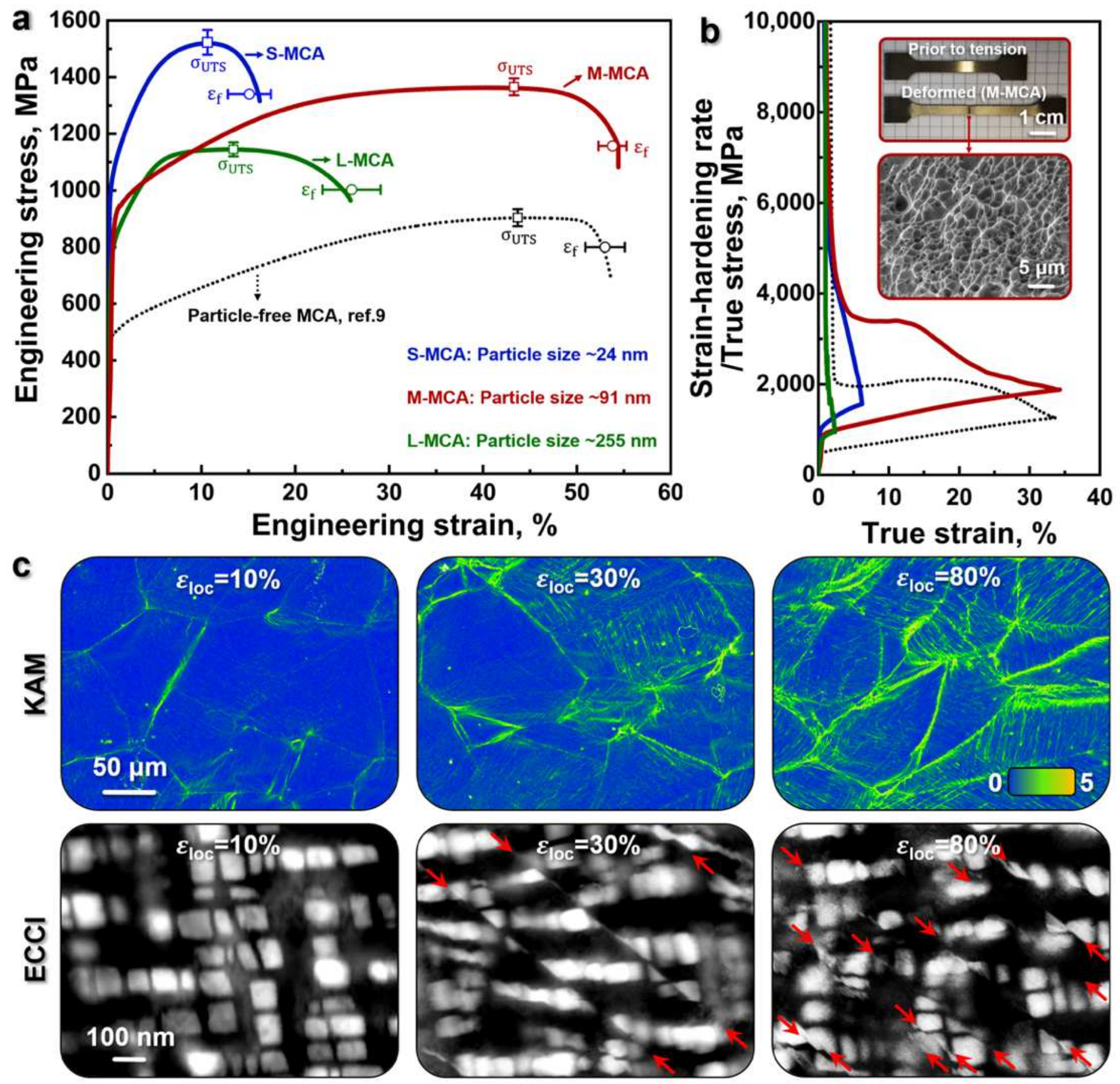

Figure 2

Mechanical behaviour and nanoscale processes during plastic straining of the M-MCA. a, Typical engineering stress-strain curves measured at room-temperature together with the average value for ultimate tensile strength ( $\sigma_{-} U T S$ ) and elongation at fracture $\left(\varepsilon_{-} f\right)$. b. Strain hardening rate/true stresstrue strain curves. The insets show the macroscopic image (upper inset) of the tensile sample and corresponding fracture morphology (bottom inset), typical ductile fracture with fine dimple structures is observed. c, Substructure evolution as a function of deformation: EBSD-KAM maps revealing the deformation-induced strain distribution (upper); $\mathrm{ECCl}$ analysis (bottom) showing the evolution of microbands, and the shearing of L12 particles are highlighted by red arrows. 

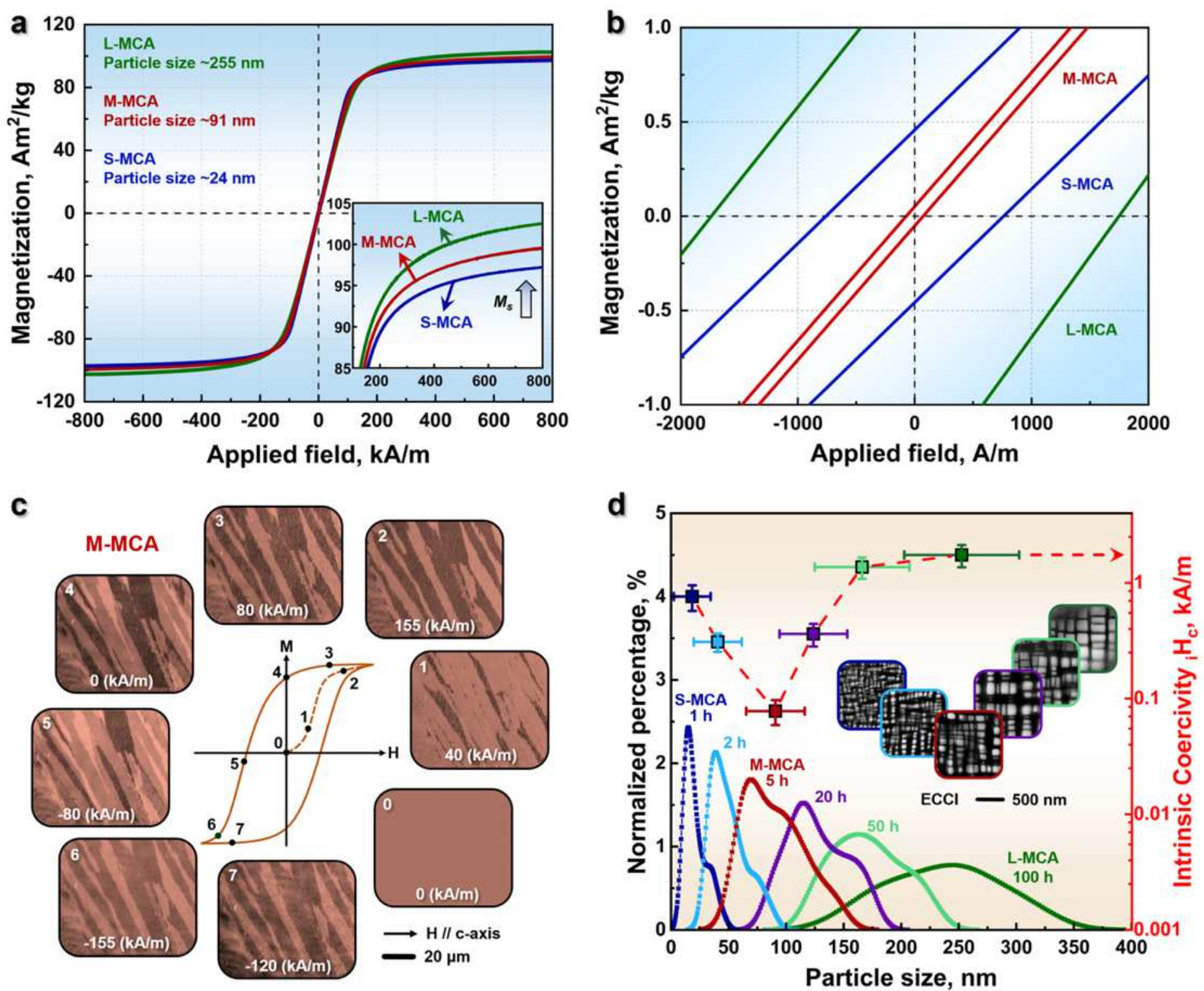

\section{Figure 3}

Soft magnetic response and associated Bloch wall motion behaviour of the MCAs at room-temperature. a, Hysteresis loops $(M / H)$ acquired up to $\pm 800 \mathrm{kA} / \mathrm{m}$. The magnetic field-sweeping rate is $1 \mathrm{kA} / \mathrm{m}$. The inset shows the enlarged view of the increase in saturation magnetization with particle coarsening. $b$, $\mathrm{M} / \mathrm{H}$ curves measured at a rate of $0.1 \mathrm{kA} / \mathrm{m}$ between $\pm 50 \mathrm{kA} / \mathrm{m}$, showing the extremely low coercivity. $\mathrm{c}$, MOKE microscopy observation, in longitudinal contrast, revealing the magnetization process. The applied magnetic field is horizontal to the viewing plane. $d$, Statistical particle size distribution of all the MCAs under different isothermal heat treatment conditions $(1 \sim 100 \mathrm{~h}$ at $1173 \mathrm{~K})$. The right inset shows the evolution of particle size by ECCl probing. 

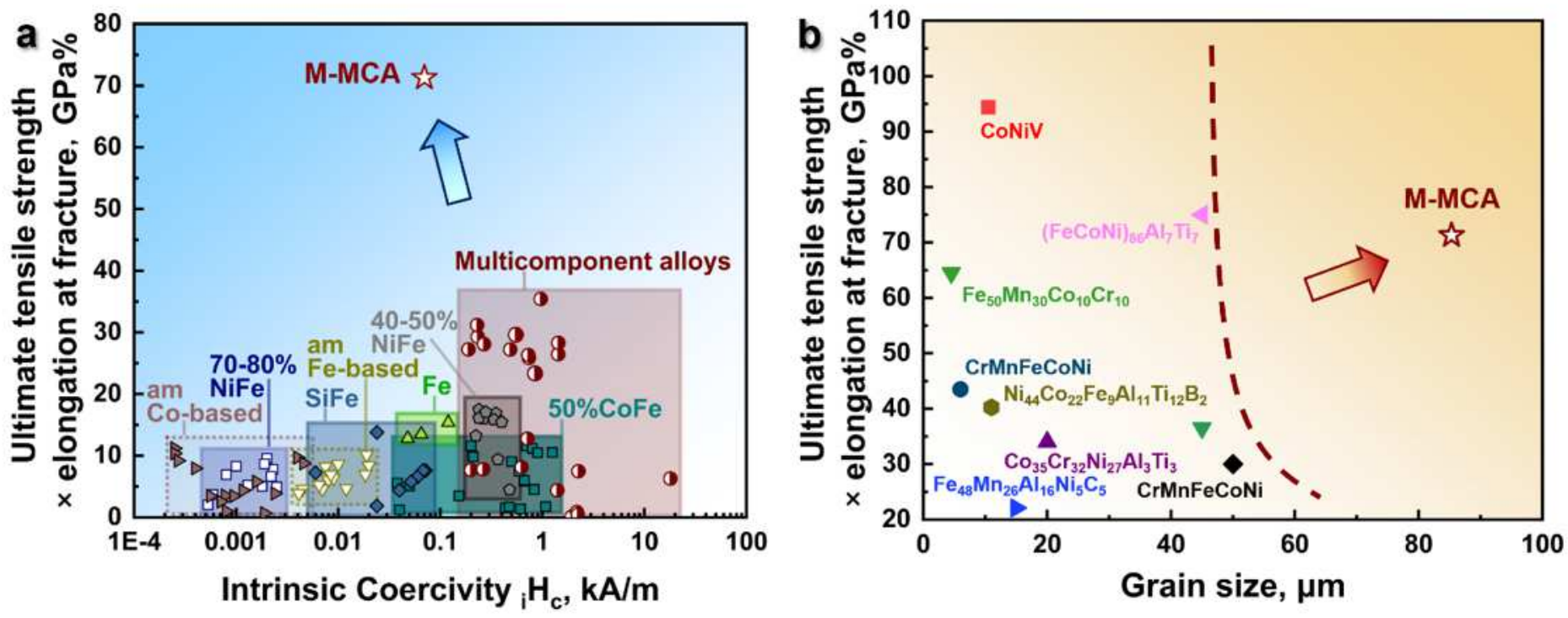

\section{Figure 4}

Mechanical and magnetic features combined in the new Fe32Co28Ni28Ta5Al7 (at.\%) M-MCA material. a, Ashby map compiling room-temperature ultimate tensile strength ( $\left.\sigma_{-} U T S\right) \times e l o n g a t i o n$ at fracture $\left(\varepsilon_{-} f\right)$ and intrinsic coercivity compared to those of other soft magnetic materials, such as NiFe29,30, CoFe31,32, SiFe33, Fe34, amorphous alloys4,35-37 and established MCAs38-49. b, Ashby map showing $\sigma \_U T S \times \varepsilon_{-} f$ versus average grain size compared to those of other strong and ductile MCAs22-24,50-53. 'am' in the legend stands for amorphous alloys.

\section{Supplementary Files}

This is a list of supplementary files associated with this preprint. Click to download.

- ExtendedData.docx 